\title{
BMJ
}

RESEARCH

\section{Peer support for patients with type 2 diabetes: cluster randomised controlled trial}

\author{
S M Smith, senior lecturer in primary care, ${ }^{1} \mathrm{G}$ Paul, research project manager, ${ }^{1} \mathrm{~A}$ Kelly, senior lecturer in \\ biomedical statistics, ${ }^{1} \mathrm{D} L$ Whitford, professor of family and community medicine, ${ }^{2} \mathrm{E}$ O'Shea, professor of \\ economics, ${ }^{3}$ T O’Dowd, professor of general practice ${ }^{1}$
}

'Department of Public Health and Primary Care, Trinity College,

Dublin, Republic of Ireland

${ }^{2}$ Department of Family and Community Medicine, Royal College of Surgeons in IrelandMedical University of Bahrain, PO Box 15503, Adliya, Bahrain

${ }^{3}$ Irish Centre for Social

Gerontology, National University of Ireland, Galway

Correspondence to: S M Smith susmith@tcd.ie

Cite this as: BMJ 2011;342:d715 doi:10.1136/bmi.d715

\section{ABSTRACT}

Objective To test the effectiveness of peer support for patients with type 2 diabetes.

Design Cluster randomised controlled.

Setting 20 general practices in the east of the Republic of Ireland.

Participants 395 patients (192 in intervention group, 203 in control group) and 29 peer supporters with type 2 diabetes.

Intervention All practices introduced a standardised diabetes care system. The peer support intervention ran over a two year period and contained four elements: the recruitment and training of peer supporters, nine group meetings led by peer supporters in participant's own general practice, and a retention plan for the peer supporters.

Main outcome measures $\mathrm{HbA}_{1 \mathrm{c}}$; cholesterol concentration; systolic blood pressure; and wellbeing score.

Results There was no difference between intervention and control patients at baseline. All practices and 85\% (337) of patients were followed up. At two year follow-up, there were no significant differences in $\mathrm{HbA}_{1 \mathrm{c}}$ (mean difference $-0.08 \%, 95 \%$ confidence interval $-0.35 \%$ to $0.18 \%$ ), systolic blood pressure $(-3.9 \mathrm{~mm} \mathrm{Hg},-8.9$ to $1.1 \mathrm{~mm} \mathrm{Hg})$, total cholesterol concentration $(-0.03 \mathrm{mmol} / \mathrm{L},-0.28$ to $0.22 \mathrm{mmol} / \mathrm{L})$, or wellbeing scores $(-0.7,-2.3$ to 0.8$)$. While there was a trend towards decreases in the proportion of patients with poorly controlled risk factors at follow-up, particularly for systolic blood pressure (52\% (87/166) >130 mm Hg in intervention v 61\% (103/169) $>130 \mathrm{~mm} \mathrm{Hg}$ in control), these changes were not significant. The process evaluation indicated that the intervention was generally delivered as intended, though $18 \%$ (35) of patients in the intervention group never attended any group meetings.

Conclusions A group based peer support intervention is feasible in general practice settings, but the intervention was not effective when targeted at all patients with type 2 diabetes. While there was a trend towards improvements of clinical outcomes, the results do not support the widespread adoption of peer support.

Trial registration Current Controlled Trials ISRCTN42541690.

\section{INTRODUCTION}

There has been an increasing focus on involvement of patients in chronic disease care, and the World Health Organization's action plan for chronic disease management encourages governments to take action to help people manage their own chronic conditions better by providing education, incentives, and tools for self management and care. ${ }^{1}$ Type 2 diabetes is a chronic disease that is rising in prevalence across the world and placing increasing demands on healthcare systems. WHO has suggested that peer support is a promising approach for diabetes care as it harnesses the ability of patients with diabetes to support each other in managing their everyday lives. ${ }^{2}$ Peer support has been defined as the provision of support from an individual with experiential knowledge based on a sharing of similar life experiences. ${ }^{3}$ It is usually provided within a volunteering framework and can be delivered in many ways, including group or individual support or through more remote formats such as telephone or internet based support.

Peer support has been used in various conditions with varied results, ${ }^{4-10}$ but there is limited evidence to support its effectiveness, particularly for people with type 2 diabetes. There is also substantial variation in the degree of training and level of involvement of the peer supporters or community health workers in these studies, with many having a predominantly educational focus and peer groups being facilitated by health professionals rather than peers themselves. ${ }^{10}$

We report the results of a pragmatic cluster randomised controlled trial examining the effectiveness of peer support in improving biophysical and psychosocial outcomes for people with type 2 diabetes. The intervention was based on social support theory and was delivered in groups based in the general practices of participating patients.

\section{METHODS}

The methods and intervention development have been reported in detail previously. ${ }^{112}$ In brief, this was a cluster randomised controlled trial set in general practices in the Republic of Ireland. Diabetes care in Ireland has generally been unstructured, with more 
than half of patients still attending specialist diabetes clinics for annual reviews. ${ }^{13}$ Thirty two practices from the Trinity College Dublin network of teaching practices were invited to participate, and 20 accepted. Practices were eligible to participate if they had a practice nurse; had computerised records; did not participate in the pilot study; had a minimum of about 1000 patients with GMS cards (eligible for free healthcare) or had more than 50 patients on their register of those with type 2 diabetes, or both; and were not participating in an existing shared care diabetes programme involving structured care between the general practices and hospital diabetes clinics. Participating practices were given a grant of $€ 5000(£ 4200, \$ 6900)$ a year for three years. This grant reflected the fact that data collection and intervention delivery were carried out at practice level with the support of the research team.

Practices were stratified by practice size and the presence of existing structured diabetes care and were then allocated to control or intervention group by an independent statistician using minimisation. ${ }^{14}$ All

\section{The peer support intervention}

The peer support intervention had the following components:

\section{Peer supporters}

Peer supporters were identified by general practitioners and practice nurses and were trained at a ratio of about one peer supporter to seven or eight patients with type 2 diabetes. The criteria for eligibility were:

- Having had type 2 diabetes for at least one year

- Participation in preventive treatments and judged by the practice team as being generally adherent to treatment and behaviour change regimens

- Capacity and commitment to undergo the training required

- A full understanding of the importance of patients' confidentiality

- Undertaking to liaise with the practice nurse or general practitioner if unanticipated problems arose during the course of their peer support activity

\section{Peer supporter training}

The peer supporters attended two evening training sessions, which were conducted by the research team. These sessions focused on the basics of type 2 diabetes and issues relating to working with groups and confidentiality.

\section{Peer support meetings}

Peer support meetings were held in the general practice premises at a convenient time for practice staff, peer supporters, and participants. Practices offered various daytime or early evening sessions, depending on patients' preference. There were nine peer support sessions over two years; at month one, month two, and every three months thereafter. Each meeting was facilitated by the peer supporter, and there were no health professionals present in the meeting room though they were available on site, if needed. Each meeting had a suggested theme and a small structured component. The contents of the meetings were recorded (see appendix 2 on bmj.com). There was also a "frequently asked questions" (FAQs) system - that is, at the end of each session the group fed back questions to the research team who compiled written answers based on the feedback from all groups. The FAQs from all groups were combined and sent back to the groups for the next session.

\section{Retention and support of peer supporters}

Formal structures were put in place to ensure peer support workers were supported in their role, including telephone calls from the project manager before and after meetings; a course handbook and resource pack; an annual social or educational event; a protocol to follow if a peer supporter resigned; and travel and related expenses (this was given in the form of a general shopping voucher at the end of each year with a value of $€ 300$ ). practices introduced a structured diabetes care system as only two of them had such systems in place before the study. This involved regular recall of patients every three to six months with an annual audit of risk factors. This was done to standardise delivery of diabetes care across all study practices so that the peer support intervention would be an addition to diabetes care. All practices created or, in the case of two practices, updated their diabetes registers. Intervention practices then recruited three individuals as peer supporters.

The box outlines the eligibility criteria for peer supporters. They attended two evening training sessions each lasting about 90 minutes and delivered by the research team. Appendix 1 on bmj.com describes the content of the training sessions. Peer supporters were advised that they were not being asked to provide healthcare or education and that the emphasis was on social support. There was also a strong emphasis on the importance of confidentiality, and peer supporters were given specific advice as to how they could seek support from their general practitioner and practice nurse or the study manager if needed. The general practitioners and practice nurses also received training in relation to the study protocol and delivering standardised diabetes care (one author (SMS) delivered a 90 minute session in each practice with an academic detailing approach). Further details of the training are provided in the study protocol. ${ }^{12}$

We used a random number list to select patients randomly from the practice diabetes register until a total of 21 patients were recruited in each practice. To be eligible patients had to be aged over 18, have type 2 diabetes, be able to participate in a group, and be attending one of the participating practices. Those who agreed to participate were invited to attend their practice to complete a questionnaire containing demographic information and study outcome measures. The practice nurse then collected biophysical outcome data.

The intervention was developed with the MRC Framework for the evaluation of complex interventions to improve health. ${ }^{15}$ The underlying theoretical framework was social support, and the full development of the intervention has been described in detail previously. ${ }^{11}$ The intervention was delivered over a two year period, from May 2007 to April 2009. The box describes the components of the intervention.

The primary outcomes were $\mathrm{HbA}_{1 \mathrm{c}}$ (measured with reversed phase cation exchange liquid chromatography, with an automatic glycol-haemoglobin analyser, DCCT compatible); blood pressure (measured with an OMRON M5-1 Automatic BP monitor); cholesterol concentration (analysed in local hospital laboratories with automated clinical chemistry analysers), and wellbeing. ${ }^{16}$ Secondary outcomes were body mass index (BMI), diabetes self care activities, ${ }^{17}$ self efficacy, ${ }^{18}$ adherence to medications, ${ }^{19}$ family and friends subscale of the chronic illness resources survey, ${ }^{20}$ smoking (self reported), prescriptions (aspirin, antihypertensive drugs, and cholesterol lowering agents), and measures of the process of care (visits 


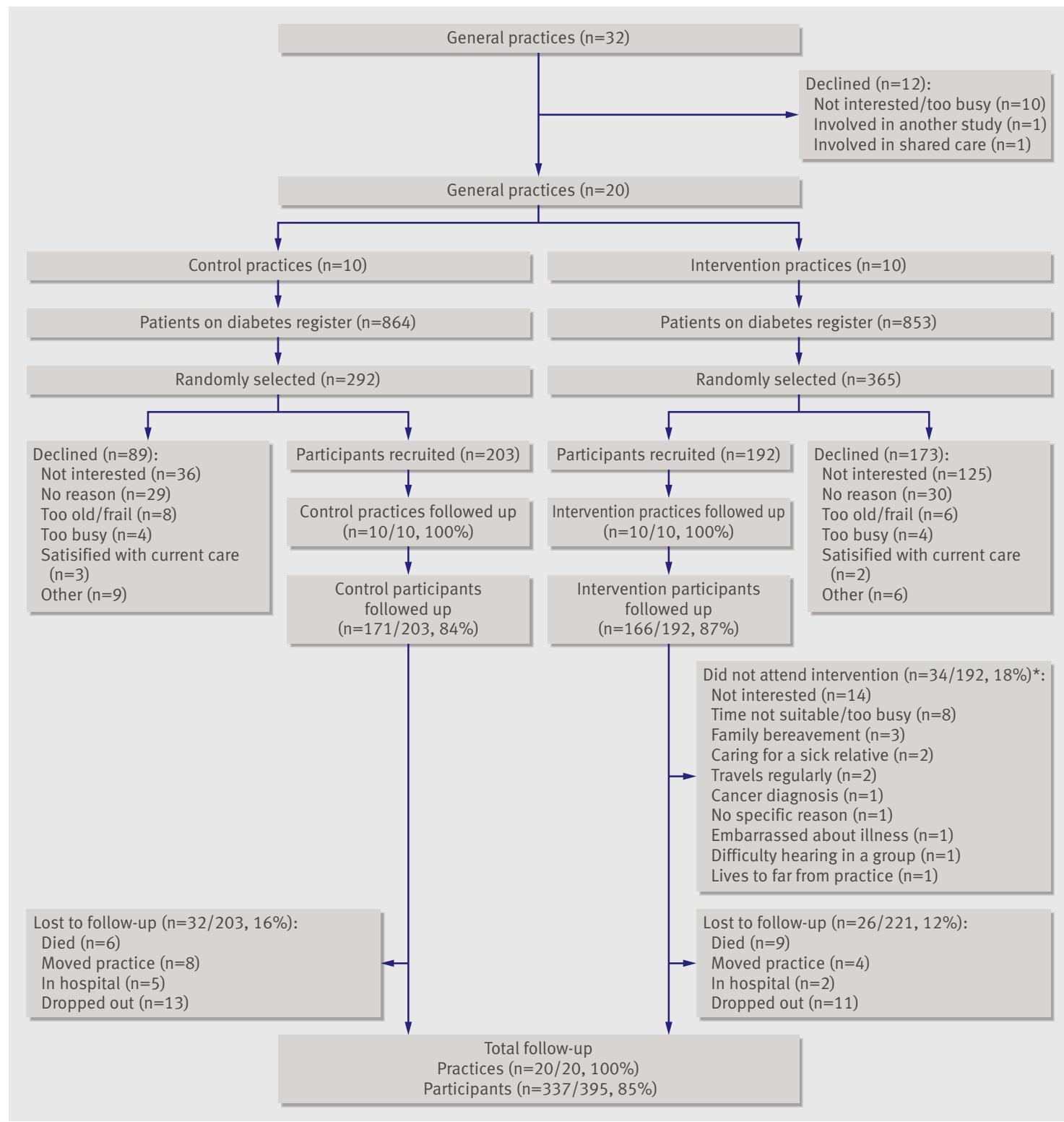

Recruitment and follow-up of practices and participants. *Does not include participants lost to follow-up

to general practitioner, practice nurse, hospital diabetes outpatients department, and hospital diabetes centre and admissions to hospital). Demographic details, including measures of socioeconomic status (medical card status) and educational attainment, were also collected. Participants completed the questionnaire while waiting to see the practice nurse, who then checked that the questionnaire was filled in and completed the biophysical measures. The nurses extracted data relating to the process of care from the patients' records and entered all data into a FileMaker Pro database. ${ }^{21}$ The project manager doubled checked the data. Full details of data collection and storage procedures are outlined in the study protocol. ${ }^{12}$ We could not blind participants or practice nurses collecting data to group allocation because of the nature of the intervention. Three of the four primary outcomes, however, were collected with automated tests.
We undertook a process evaluation of the trial and measured treatment fidelity using a framework developed by Bellg et al. ${ }^{22}$ This involved consideration of five elements: treatment design, training procedures, delivery of treatment, receipt of treatment, and enactment of treatment skills. This information was collected with peer supporter log diaries, the study managers' contact records, and focus groups with practice staff, peer supporters, and participants. Parallel qualitative and economic analyses are ongoing and will be reported elsewhere.

\section{Sample size calculation}

We aimed to achieve a sample of 400 patients from 20 practices. This incorporated the effect of cluster randomisation and allowed for 80-85\% follow-up of patients and a $15 \%$ rate of practice attrition. Sample size calculations also incorporated a 20\% improvement from 
Table 1|Baseline characteristics of participants with type 2 diabetes allocated to peer support (intervention) or no peer support (control) and peer supporters. Figures are numbers (percentage) of participants unless stated otherwise

Intervention group $(n=192)$
Control group $(n=203)$

Peer supporters $(n=29)$

Practice factors at baseline

\begin{tabular}{|c|c|c|c|}
\hline No of practices & 10 & 10 & - \\
\hline Mean No of patients/practice & 4830 & 5800 & - \\
\hline Urban location & 8 & 8 & - \\
\hline $\begin{array}{l}\text { Mean \% of practice population on diabetes } \\
\text { register }\end{array}$ & $1.7 \%$ & $1.7 \%$ & - \\
\hline \multicolumn{4}{|l|}{ Diabetes care*: } \\
\hline GP only & $68(35)$ & $60(30)$ & - \\
\hline GP and specialist & $60(30)$ & $73(36)$ & - \\
\hline Specialist only & $62(32)$ & $65(32)$ & - \\
\hline No care & $3(2)$ & $5(2)$ & - \\
\hline \multicolumn{4}{|l|}{ Participants and peer supporters } \\
\hline Women & $88(46)$ & $93(46)$ & $17(59)$ \\
\hline Mean (SD) age (years) $(n=424)$ & $66.1(11.11)$ & $63.2(11.04)$ & $62.7(11.3)$ \\
\hline Mean (SD) duration of diabetes (years) $(n=418)$ & $7.4(7)$ & $6.9(6.3)$ & $6.8(8.1)$ \\
\hline GMS card $†$ & $92(48)$ & $108(53)$ & $8(30)$ \\
\hline \multicolumn{4}{|l|}{ Education status: } \\
\hline Primary education only & $79(41)$ & $98(48)$ & $5(17)$ \\
\hline Complete third level education & $15(8)$ & $10(5)$ & $9(31)$ \\
\hline Self reported smoking & $31(16)$ & $40(20)$ & $4(14)$ \\
\hline \multicolumn{4}{|l|}{ Diabetes regimen: } \\
\hline Diet controlled & $52(27)$ & 38 (19) & $9(31)$ \\
\hline Oral hypoglycaemic drugs & $133(69)$ & $160(79)$ & $14(48)$ \\
\hline Insulin & $5(3)$ & $3(1)$ & $2(7)$ \\
\hline Missing & $2(1)$ & $2(1)$ & $4(14)$ \\
\hline \multicolumn{4}{|l|}{ Marital status: } \\
\hline Married/cohabiting & $134(68)$ & $113(59)$ & $20(70)$ \\
\hline Single, widowed, separated, divorced & $65(33)$ & $79(41)$ & $9(30)$ \\
\hline Missing & $2(0.5)$ & $0(0)$ & $0(0)$ \\
\hline$\geq 3$ medical conditions & $134(70)$ & $129(64)$ & $27(93)$ \\
\hline
\end{tabular}

*According to patient.

†Eligibility for GMS (general medical services) card implies being in 30\% of population with lowest income and indicates eligibility for free healthcare.
$<5 \mathrm{~mol} / \mathrm{L}$, which was the treatment target for cholesterol at that time. ${ }^{25}$ Locally available data indicated that $57 \%$ of patients had a concentration $>5 \mathrm{mmol} / \mathrm{L}$ and the intracluster coefficient was $0.06 .^{10}$

Wellbeing scores-We needed 221 patients from 12 practices to show a clinically significant difference in wellbeing scores between intervention and control groups (that is, a mean difference of 5 points, SD 10.3 ; intracluster coefficient $0.07^{24}$ ).

\section{Statistical analysis}

The analyses were based on intention to treat and are reported according to the CONSORT guidelines for the reporting of cluster randomised controlled trials. ${ }^{26}$ We also undertook a sensitivity analysis and a per protocol analysis to estimate the effect of exposure to the intervention or group attendance on outcomes. Preplanned analyses were also conducted to examine those participants whose risk factors were above target ranges at baseline - that is, $\mathrm{HbA}_{1 \mathrm{c}}>7 \%$, systolic blood pressure $>130 \mathrm{~mm} \mathrm{Hg}$, and cholesterol concentration $>4.8 \mathrm{mmol} / \mathrm{L}$. These were the targets presented to the clinicians involved in delivering diabetes care across both intervention and control practices.

We used multilevel linear or logistic regression models with random effects of patients nested within practices. In these models, the primary fixed effect of interest is the differential effect of intervention versus control over time. For the subgroup analysis, we selected individuals deemed out of control at baseline for modelling with practice as a random effect. The effect size in this instance refers to the contrast between the intervention versus the control group at follow-up. For all models, we included additional patient specific (such as age and sex) and practice specific (such as type of practice) covariates. The multilevel analysis was conducted with $\mathrm{R}(2.11) .{ }^{27}$

Analysis of secondary outcomes was limited to an intention to treat cluster level analysis apart from analysis of BMI, which achieved a significant effect in this preliminary analysis so was then entered into the multilevel model analysis.

the intervention group. All calculations were two sid and based on an $\alpha$ of $5 \%$ and a power of $80 \%$. Full details are reported in the study protocol. ${ }^{12}$

$H b A_{1 c}-$ We needed 130 patients from eight practices to show a clinically significant difference in mean $\mathrm{HbA}_{1 \mathrm{c}}$ between intervention and control groups (that is, a difference of $0.9 \%{ }^{23}$; SD 1.6 , intracluster coefficient $0.001^{24}$ ).

Systolic blood pressure-We needed 400 patients from 20 practices to show a significant improvement in the proportion of patients with a systolic blood pressure below $160 \mathrm{~mm} \mathrm{Hg}$. This was in the context of a treatment target of $135 \mathrm{~mm} \mathrm{Hg},{ }^{25}$ based on locally available data indicating that $46 \%$ of patients in a previous study in Dublin had a systolic blood pressure $>160 \mathrm{~mm} \mathrm{Hg}$ and the intracluster coefficient was $0.001 .^{24}$

Cholesterol -We needed 410 patients from 20 practices to show a significant improvement in the proportion of patients with a cholesterol concentration

\section{RESULTS}

The figure shows the flow of practices and patients through the study. We could not carry out baseline data collection before randomisation of practices as we had to first identify intervention practices so that we could recruit and train the peer supporters, which took place over a six month period. As a result, there was a difference in recruitment rates of patients between intervention and control practice. More potential participants had to be invited to participate in the study in intervention practices. Recruitment of participants took place between November 2006 and April 2007 and the intervention ran from May 2007 until April 2009. Non-participants in both the intervention and control practices were similar in terms of sex, age, and socioeconomic status (as measured by 
Table $2 \mid$ Primary outcomes at baseline and follow-up in participants with type 2 diabetes allocated to peer support (intervention) or no peer support (control)

\begin{tabular}{|c|c|c|c|c|c|c|c|c|c|}
\hline & \multicolumn{2}{|c|}{$\begin{array}{c}\text { No of people (baseline/ } \\
\text { follow-up) }\end{array}$} & \multicolumn{2}{|c|}{$\begin{array}{l}\text { Mean (SD) outcome } \\
\text { at baseline }\end{array}$} & \multicolumn{2}{|c|}{$\begin{array}{l}\text { Mean (SD) outcome } \\
\text { at follow-up }\end{array}$} & \multirow[b]{2}{*}{ ICC } & \multirow[b]{2}{*}{ Mean difference $(95 \% \mathrm{Cl})$} & \multirow[b]{2}{*}{ Pvalue } \\
\hline & Intervention & Control & Intervention & Control & Intervention & Control & & & \\
\hline $\mathrm{HbA}_{1 \mathrm{c}}(\%)$ & $187 / 165$ & $201 / 170$ & $7.2(1.4)$ & $7.2(1.2)$ & $7.1(1.1)$ & $7.1(1.2)$ & 0.005 & $-0.08(-0.35$ to 0.18$)$ & 0.64 \\
\hline Systolic blood pressure $(\mathrm{mm} \mathrm{Hg})$ & $192 / 166$ & $202 / 169$ & $146(21)$ & $144(18)$ & $136(19)$ & 137 (15) & 0.007 & $-3.9(-8.9$ to 1.1$)$ & 0.12 \\
\hline Cholesterol (mmol/L) & $186 / 164$ & $201 / 170$ & $4.1(0.9)$ & $4.5(1.2)$ & $3.9(0.9)$ & $4.3(0.9)$ & 0.004 & $-0.03(-0.28$ to 0.22$)$ & 0.81 \\
\hline Wellbeing score* & $192 / 147$ & 201/157 & $25.0(6.8)$ & $23.9(7.6)$ & $23.7(5.1)$ & $23.2(5.8)$ & 0.0001 & $-0.71(-2.3$ to 0.8$)$ & 0.36 \\
\hline
\end{tabular}

eligibility for free primary health care). Follow-up data collection took place between May and June 2009.

Table 1 gives the characteristics of patients in the intervention and control group and the peer supporters. Tables 2 and 3 present the primary and secondary outcomes. They indicate better than anticipated control of risk factors at baseline, though a considerable proportion of patients still had risk factors above target levels. Of the participants, 163/388 (42\%) had an $\mathrm{HbA}_{1 \mathrm{c}}$ above 7\%; 291/394 (74\%) had a systolic blood pressure above $130 \mathrm{~mm} \mathrm{Hg}$, and 89/387 (23\%) had a total cholesterol concentration above $4.8 \mathrm{mmol} / \mathrm{L}$. These treatment targets for risk factors differed from those used for the original power calculation as there had been changes in the target levels in the intervening years.

\section{Follow-up results}

At follow-up we found no significant improvements in any primary or secondary outcomes when we used multi-level modelling that accounted for clustering and other confounding variables (see tables 2 and 3 ). We also carried out multi-level modelling to examine the subgroups of patients with poorly controlled risk factors at baseline, and, while there was a trend towards clinically relevant improvements in proportions with better blood pressure control, these effects were not significant (table 4).

We carried out additional per protocol analyses as planned to test whether there were any links between group attendance and outcomes. We found nothing relating to attendance versus non-attendance and to numbers of groups attended by participants that could be regarded as a dose effect.

\section{Peer supporters}

At baseline data from peer supporters and participants were similar (table 1), though peer supporters had attained a higher level of education. Primary outcomes at baseline for the peer supporters were mean $\mathrm{HbA}_{1 \mathrm{c}}$ $6.8 \%$, mean total cholesterol $4.3 \mathrm{mmol} / \mathrm{L}$, mean systolic blood pressure $140 \mathrm{~mm} \mathrm{Hg}$, and mean wellbeing score 27. Primary outcomes at follow-up were mean $\mathrm{HbA}_{1 \mathrm{c}} 6.9 \%$, mean total cholesterol $3.7 \mathrm{mmol} / \mathrm{L}$, mean systolic blood pressure $139 \mathrm{~mm} \mathrm{Hg}$, and mean wellbeing score 24 . Secondary outcomes at baseline and follow-up were also similar in peer supporters and participants. Twenty nine (97\%) peer supporters were followed up. The descriptive analysis of the peer supporters at follow-up indicated no significant changes over time apart from some decline in wellbeing (mean score 27 at baseline; 24.1 at follow-up).

\section{Process evaluation}

The training and intervention were delivered as planned for the general practitioners, practice nurses, and peer supporters in the protocol. All intervention and control practices implemented structured diabetes care as planned. All the practices and 28 out of the 29 peer supporters were followed up, though only 23 of the peer supporters were retained in their role. The main concern regarding the delivery and receipt of treatment - that is, the intervention-was the low attendance at the group meetings. Participants in the intervention group attended a mean of five peer support meetings, and 18\% never attended a meeting and therefore had no exposure to the intervention. This was despite repeated phone calls from practice nurses and a call from the study manager to all non-attenders after the third round of meetings.

Peer supporters were contacted after each meeting and also kept diaries. Appendix 2 on bmj.com provides data collected about the content of peer support meetings. In general, the groups followed and discussed the planned topics.

The process evaluation also highlighted the heavy workload involved in delivering a peer support intervention over two years. There was a mean of 15 contacts between the study manager and the intervention practices relating specifically to the peer support intervention rather than the research process. There was a mean of 25 contacts with the peer supporters during the two year period. These contacts included training sessions, meetings, telephone calls, and letters and indicate that as an intervention peer support requires substantial clinical and administrative input. Most of these contacts related to running the intervention rather than collection of research data.

\section{DISCUSSION}

For people with diabetes a group based peer support intervention is feasible in general practice settings. While there was a trend towards improved management of clinical risk factors, however, peer support did not significantly improve physical and psychosocial outcomes for people with type 2 diabetes. One 
Table 3 |Secondary outcomes at baseline and follow-up in participants with type 2 diabetes allocated to peer support (intervention) or no peer support (control). Figures are mean (SD) scores except where indicated

\begin{tabular}{|c|c|c|c|c|c|c|c|c|}
\hline \multirow[b]{2}{*}{ Variable } & \multicolumn{2}{|c|}{$\begin{array}{c}\text { No of people } \\
\text { (baseline/follow-up) }\end{array}$} & \multicolumn{2}{|c|}{ Outcome at baseline } & \multicolumn{2}{|c|}{ Outcome at follow-up } & \multirow{2}{*}{$\begin{array}{c}\text { Mean } \\
\text { difference }\end{array}$} & \multirow[b]{2}{*}{ Pvalue } \\
\hline & Intervention & Control & Intervention & Control & Intervention & Control & & \\
\hline BMI & $191 / 164$ & $200 / 168$ & $30.3(1.5)$ & $31.8(1.2)$ & $30.3(1.5)$ & $31.7(1.1)$ & 1.4 & $0.56^{*}$ \\
\hline MARS score $†$ & $184 / 157$ & $197 / 163$ & $23.9(1.7)$ & $23.8(1.8)$ & $24.1(0.3)$ & $23.9(0.4)$ & 0.2 & 0.13 \\
\hline Social support scoref & $192 / 148$ & $199 / 152$ & $23.8(9.2)$ & $22.4(8.4)$ & $23.6(2)$ & $23(4.3)$ & 0.6 & 0.7 \\
\hline DMSES§ & $191 / 146$ & $201 / 137$ & $119(26.6)$ & $112(26.5)$ & $117.1(12.1)$ & $117.5(8.1)$ & -0.4 & 0.9 \\
\hline \multicolumn{9}{|l|}{ SDSCA scoreף: } \\
\hline General diet & \multirow{5}{*}{$192 / 154$} & \multirow{5}{*}{$201 / 156$} & $5.1(2.1)$ & $4.7(2.1)$ & $5.1(1.8)$ & $5.2(1.8)$ & -0.1 & 0.9 \\
\hline Specific diet & & & $4.2(1.8)$ & $4.1(1.7)$ & $4.3(1.5)$ & $4.1(1.5)$ & 0.2 & 0.3 \\
\hline Foot care & & & $2.7(2.4)$ & $2.7(2.4)$ & $3(2.5)$ & $2.9(2.2)$ & 0.1 & 0.5 \\
\hline Exercise & & & $3(2.5)$ & $2.7(2.3)$ & $3.3(2.2)$ & $3.5(2.5)$ & -0.2 & 0.8 \\
\hline Blood glucose testing & & & $4.8(2.7)$ & $4(2.9)$ & $4.9(2.6)$ & $4.3(2.9)$ & 0.6 & 0.1 \\
\hline $\begin{array}{l}\text { No (\%) who self reported } \\
\text { smoking }\end{array}$ & $186 / 161$ & $202 / 168$ & 31 (16) & $40(20)$ & $25(15.5)$ & 29 (17) & $1.5 \%$ & 0.7 \\
\hline \multicolumn{9}{|c|}{$\begin{array}{l}\text { *Adjusted with multilevel modelling as } \mathrm{P}=0.03 \text { in simple cluster level analysis. } \\
\text { †Medication adherence score, range } 5-25 \text {, higher scores=better adherence. } \\
\text { †Range } 8-40 \text {, higher scores=more social support experienced. } \\
\text { §Diabetes management self efficacy scale, range } 0-150 \text { ( }(50 \text { low, } 50-100 \text { medium, } 100-150 \text { high). } \\
\text { ॠSummary of self care activities score, range } 0-7 \text {, higher score=higher level of self care. }\end{array}$} \\
\hline
\end{tabular}

explanation for this is that, despite the hope that peers could be harnessed to improve outcomes for patients, peer support is ineffective when delivered as a structured intervention. There are some features of this study, however, that suggest we do not yet have a definitive answer about the potential effectiveness of peer support in type 2 diabetes. In particular, while the study achieved its proposed sample size, baseline levels of risk factors for diabetes had improved in the intervening years, leaving little room for improvement in mean $\mathrm{HbA}_{1 \mathrm{c}}$, systolic blood pressure, and total cholesterol concentration. The treatment targets had also changed, however, becoming tighter over time, and there were still significant proportions of patients not meeting these targets, particularly for systolic blood pressure.

Secondary outcome measures were also no different between intervention and control groups. These indicated high levels of adherence to treatment drugs and self efficacy and medium level scores for diabetes self care activities and social support at baseline and followup. The intervention might not have been intensive enough to affect broader outcomes. We were, however, trying to achieve a balance between overburdening the peer supporters with frequent meetings and having sufficient exposure to the intervention in terms of meeting intensity.

There was a non-significant reduction in wellbeing in the intervention group, and, while this is also clinically relevant, it is important to consider that peer support could have a detrimental impact on wellbeing if groups focused on negative experiences. In relation to social support, it was difficult to find a measure that reflected the type of social support that a group based intervention might provide. The measure used might have failed to detect the type of social support that peers provide compared with the support provided by family and friends, which existing measures consider. This is possible as the qualitative analysis indicated that participants valued the meetings and were positive about the support they had received from their leaders and fellow group members (G Paul, personal communication). An alternative social support outcome measure would be the Lubben social network scale. ${ }^{28}$ While this scale does focus on broader social, less disease oriented social support networks, it has been used only in older patients.

\section{Comparison with other studies}

Our results are consistent with those of other studies published on peer support for type 2 diabetes, which have also failed, in general, to show a significant impact on glycaemic control as measured by $\mathrm{HbA}_{1 \mathrm{c}}{ }^{91029-32}$ Lorig et al recently published a randomised controlled trial of their chronic disease self management programme adapted for people with diabetes. ${ }^{9}$ While they found improvements in depression and in healthy eating, there was no effect on $\mathrm{HbA}_{1 \mathrm{c}}$. Studies examining peer support have not specifically targeted patients with poorly controlled type 2 diabetes, and such patients might benefit most from a peer support intervention. Our qualitative analysis also indicated that participants thought they would have benefited from peer support around the time of diagnosis (G Paul, personal communication). The logistics of running a trial of a peer support intervention for people with a new diagnosis would be more challenging but perhaps worth pursuing.

While most of the studies of peer support for type 2 diabetes to date have shown no benefit for participants in terms of glycaemic control, benefits in terms of personal gains (training and satisfaction of helping people) for peer supporters have been reported. ${ }^{33}$ In this study, however, the peer supporters showed some decline in wellbeing at follow-up, though this might be a chance finding as numbers were small. This raises concerns 
Table 4 | Risk factors above target at baseline and follow-up in participants with type 2 diabetes allocated to peer support (intervention) or no peer support (control)

\begin{tabular}{|c|c|c|c|c|c|c|}
\hline \multirow[b]{2}{*}{ Risk factors } & \multicolumn{2}{|c|}{ No (\%) at baseline } & \multicolumn{2}{|c|}{ No (\%) at follow-up } & \multirow[b]{2}{*}{ Odds ratio $(95 \% \mathrm{Cl})$} & \multirow[b]{2}{*}{$P$ value } \\
\hline & Intervention & Control & Intervention & Control & & \\
\hline $\mathrm{HbA}_{1 \mathrm{c}}>7 \%$ & $76 / 187(41)$ & $87 / 201(43)$ & $71 / 165(43)$ & $72 / 170(42)$ & 1.84 (0.60 to 5.59$)$ & 0.28 \\
\hline Systolic BP >130 mm Hg & $143 / 192(74)$ & $148 / 202(73)$ & $87 / 166(52)$ & $103 / 169(61)$ & $0.43(0.15$ to 1.17$)$ & 0.09 \\
\hline Cholesterol >4.8 mmol/L & $30 / 186(16)$ & 59/201 (29) & 25/164 (15) & 42/170 (24) & 0.25 (0.05 to 1.34$)$ & 0.09 \\
\hline
\end{tabular}

that the role of peer supporter could be demanding and stressful for the peer supporters themselves, particularly if participants fail to attend group meetings. This has been described previously in other peer support settings. ${ }^{34}$

\section{Strengths and limitations}

This cluster randomised controlled trial of group peer support for patients with type 2 diabetes included detailed analysis of the peer supporters as well as participants. Follow-up of practices and participants was high, and there was clearly defined structured diabetes care across both intervention and control practices so that changes in diabetes care could not have been attributed to differences in healthcare delivery as we were trying to assess the potential effectiveness of the peer support intervention itself. A full process evaluation was carried out that indicated the intervention was largely delivered as planned. It also highlighted the considerable workload involved in supporting the intervention. The differential recruitment rate between intervention and control practices and the lower than expected attendance rates indicate that group based peer support is not attractive to all patients with type 2 diabetes and reduced the external validity of this study. ${ }^{35}$ Other modes of provision of peer support might need to be offered in parallel with group meetings. It might also be important to consider flexible approaches to providing peer support, such as having drop-in groups available when patients need support rather than providing scheduled courses that take no account of individual needs. ${ }^{36}$ Other issues relating to external generalisability include resourcing of practices. We provided a grant to practices to recognise the work involved in setting up peer support groups and providing ongoing informal support to the peer supporters. The workload of the study manager was

\section{WHAT IS ALREADY KNOWN ON THIS TOPIC}

Peer support could be a promising approach for diabetes care as it harnesses the ability of patients with diabetes to support each other in managing their everyday lives

There is limited evidence to date supporting its effectiveness

\section{WHAT THIS STUDY ADDS}

Though peer support group meetings can be introduced in general practice settings, many patients were not interested in participating, and $18 \%$ of those who agreed to participate never attended any meetings

There was a trend towards improvements in clinical care but no significant improvements in diabetes or psychosocial outcomes considerable and that role is essential to the running of a peer support intervention and needs to be considered if peer support is to be introduced on a larger scale.

One limitation was our inability to conceal allocation. Practices had to be randomised before we collected baseline data so that peer supporters could be identified and trained in intervention practices. This reduced the internal validity of the study as did the lack of blinding of outcome data. Practice nurses collected outcome data and so were not blind to group allocation. For three of the four primary outcomes, however, data were collected with automated tests or devices so this minimised the risk of detection bias. The study performed well in relation to the other features of internal validity for cluster randomised trials described by Eldridge et al. ${ }^{35} \mathrm{~A}$ further potential limitation is that the presence of a peer support intervention within a practice could have motivated the entire team to provide better diabetes care. The process evaluation and the data relating to prescribing, however, did not suggest differences in delivery of diabetes care between intervention and control practices.

\section{Conclusion}

This cluster randomised controlled trial indicates that it is feasible to implement a peer support system for patients with type 2 diabetes attending general practices, though not all patients will be interested in participating. The intervention was not effective in improving biophysical and psychosocial outcomes for individuals with type 2 diabetes, when targeted at all such patients. While there was a trend towards improvements of clinical outcomes, particularly for systolic blood pressure, our results suggest that peer support should not be widely adopted in clinical practice until further research is carried out. The Peers for Progress organisation is currently carrying out several trials and demonstration projects of peer support for type 2 diabetes across the world. ${ }^{37}$ Future research could focus on alternative models of delivering support or targeting support to those with poorly controlled risk factors.

The peer support study team also includes D Handy, K Keogh, M D’Eath, P Gillespie, and F O'Kelly. We thank the peer supporters, patients, and the general practitioners and practice nurses from all the participating practices. We also thank Scott Walkin, who contributed to the initial design of the study, and Graham Watt, who acted as an external reviewer for our grant and provided valuable feedback throughout.

Contributors: SMS, DLW, EO'S, and TO'D conceived the study and together with GP and AK participated in the design of the trial and intervention. All authors participated in the acquisition and analysis of 
data and in critical revision of the manuscript and have seen and approved the final version. SMS is guarantor.

Funding: This study was fully funded by the Health Research Board of Ireland (Strategic Health Research and Development Research Awards 2004, S/A 009).

Details of the role of study sponsors: The study sponsors funded the study and carried out annual and mid-term reviews of the study progress They had no part in the design of the study; the collection, analysis, and interpretation of the data; the writing of the report; and the decision to submit the article for publication.

Competing interests: All authors have completed the Unified Competing Interest form at www.icmje.org/coi_disclosure.pdf (available on request from the corresponding author) and declare: no support from any organisation for the submitted work; no financial relationships with any organisations that might have an interest in the submitted work in the previous three years, no other relationships or activities that could appea to have influenced the submitted work

Ethical approval: This study was approved by the research ethics committee of the Irish College of General Practitioners and informed written consent was given by all patients and peer supporters. Data sharing: Additional data can be obtained from the corresponding author for the purposes of secondary research.

1 World Health Organization. 2008-2013 action plan for the global strategy for the prevention and control of noncommunicable diseases. WHO, 2008.

2 Caro JF, Fisher EB. A solution might be within people with diabetes themselves. Fam Pract 2010;27(suppl 1):i1-2S.

3 Dennis CL. Peer support within a health care context: a concept analysis. Int J Nurs Stud 2003;40:321-32.

4 Dale J, Caramlau I, Lindenmeyer A, Williams SM. Peer support telephone calls for improving health. Cochrane Database Syst Rev 2009;4:CD006903.

5 Dennis CL, Hodnett E, Gallop R, Chalmers B. The effect of peer support on breast-feeding duration among primiparous women: a randomised controlled trial. Can Med Assoc J 2002;166:21-8.

6 Doull M, O'Connor AM, Robinson V, Tugwell P, Wells GA. Peer suppor strategies for improving the health and well-being of individuals with chronic diseases. Cochrane Database Syst Rev 2005;3:CD005352.

7 Manandhar DS, Osrin D, Shrestha BP, Mesko N, Tumbahanghe KM, Tamang S, et al. Effect of a participatory intervention with women's groups on birth outcomes in Nepal: cluster-randomised controlled trial. Lancet 2004;364:970-8.

8 Stephenson JM, Strange V, Forrest S, Oakley A, Copas A, Allen E, et al. Pupil-led sex education in England (RIPPLE study): clusterrandomised intervention trial. Lancet 2004;364:338-46.

9 Lorig K, Ritter PL, Villa FJ, Armas J. Community-based peer-led diabetes self-management: a randomized trial. Diabetes Educ 2009;35:641-51.

10 Norris SL, Chowdhury FM, Van Le K, Horsley T, Brownstein JN, Zhang X, et al. Effectiveness of community health workers in the care of persons with diabetes. Diabet Med 2006;23:544-56.

11 Paul G, Smith SM, Whitford D, O'Kelly F, O'Dowd T. Development of a complex intervention to test the effectiveness of peer support in type 2 diabetes. BMC Health Serv Res 2007;7:136.

12 Paul G, Smith SM, Whitford D, O'Shea E, O’Kelly F, O’Dowd T. Peer support in type 2 diabetes: a randomised controlled trial in primary care with parallel economic and qualitative analyses: pilot study and protocol. BMC Fam Pract 2007;8:45.

13 Smith S. Primary care diabetes in the Republic of Ireland. Prim Care Diabetes 2007;1:207-8.

14 Altman DG, Bland JM. Treatment allocation by minimisation. BMJ 2005;330:843.

15 Campbell NC, Murray E, Darbyshire J, Emery J, Farmer A, Griffiths F, et al. Designing and evaluating complex interventions to improve health care. BMJ 2007;334:455-9.
16 Bradley C. Handbook of psychology and diabetes. Psychology Press, 1994:89-110.

17 Toobert D, Hampson S, Glasgow R. The summary of diabetes selfcare activities measure. Diabetes Care 2000;23:943-50.

18 McDowell J, Courtney M, Edwards H, Shortridge-Baggett L. Validation of the Australian/English version of the diabetes management selfefficacy scale. Int J Nurs Stud 2005;11:177-84.

19 Horne R, Weinman J. Patients' beliefs about prescribed medicines and their role in adherence to treatment in chronic physical illness. J Psychosom Res 2001;47:555-67.

20 Glasgow R, Toobert D, Barreera M, Strycker L. The chronic illness resources survey: cross validation and sensitivity to intervention. Health Educ Res 2005;20:402-9.

21 FileMaker Pro 8. File Maker, 2005.

22 Bellg A, Resnick B, Minicucci D, Ogedegbe G, Ernst D, Borrelli B, et al. Enhancing treatment fidelity in health behaviour change studies: best practices and recommendations from the NIH behaviour change consortium. Health Psychol 2004;5:443-51.

23 UK Prospective Diabetes Study Group. Intensive blood-glucose control with sulphonylureas or insulin compared with conventional treatment and risk of complications in patients with type 2 diabetes (UKPDS 33). Lancet 1998;352:837-53.

24 Smith SM, Bury G, O’Leary M, Shannon W, Tynan A, Staines A, et al. The North Dublin randomized controlled trial of structured diabetes shared care. Fam Pract 2004;21:39-45.

25 O'Sullivan T, Harkins V, Houlihan J. Guidelines for diabetes care in the community. Irish College of General Practitioners, 2000.

26 Campbell M, Elbourne D, Altman D. CONSORT statement: extension to cluster randomised trials. BM/ 2004;328:702-8.

27 R Development Core Team. R: a language and environment for statistical computing. Multilevel models using package LME4. $R$ Foundation for Statistical Computing, 2010.

28 Lubben J, Gironda M. Measuring social networks and assessing their benefits. In: Phillipson C, Allan G, Morgan D, Ashgate D, eds. Social networks and social exclusion: sociological and policy perspectives. Ashgate Publishing, 2004.

29 Baksi AK, Al-Mrayat M, Hogan D, Whittingstall E, Wilson P, Wex J. Peer advisers compared with specialist health professionals in delivering a training programme on self-management to people with diabetes: a randomized controlled trial. Diabet Med 2008;25:1076-82.

30 Cade JE, Kirk SFL, Nelson P, Hollins L, Deakin T, Greenwood DC, et al. Can peer educators influence healthy eating in people with diabetes? Results of a randomised controlled trial. Diabet Med 2009;26:1048-54.

31 Keyserling TC, Samuel-Hodge CD, Ammerman AS, Ainsworth BE, Henriquez-Roldan CF, Elasy TA, et al. A randomized trial of an intervention to improve self-care behaviors of African-American women with type 2 diabetes-impact on physical activity. Diabetes Care 2002;25:1576-83.

32 Lorig K, Ritter P, Villa F, Piette J. Spanish diabetes self-management with and without automated telephone reinforcement. Diabetes Care 2008;31:408-14.

33 Struthers R, Hodge FS, De Cora L, Geishirt-Cantrell B. The experience of native peer facilitators in the campaign against type 2 diabetes. I Rural Health 2003;19:174-80.

34 Cupples ME, Stewart MC, Percy A, Hepper P, Murphy C, Halliday HL. An RCT of peer-mentoring for first-time mothers in socially disadvantaged areas (the MOMENTS Study). Arch Dis Childhood 2010 June 3 [epub ahead of print].

35 Eldridge S, Ashby D, Bennett C, Wakelin M, Feder G. Internal and external validity of cluster randomised trials: systematic review of recent trials. BMJ 2008;336:876-80.

36 Funnell MM. Peer-based behavioural strategies to improve chronic disease self-management and clinical outcomes: evidence, logistics, evaluation considerations and needs for future research. Fam Pract 2010;27(suppl 1):i17-22S

37 Peers for progress. 2010. www.peersforprogress.com/.

Accepted: 15 November 2010 